\title{
ASPECTOS COGNITIVO-EMOTIVOS EN VÍCTIMAS Y AGRESORES DEL BULLYING.
}

\author{
COGNITIVE AND EMOTIONAL ASPECTS PRESENTED IN VICTIMS OF VICTMS AND \\ PERPETRATORS OF BULLYING
}

Ana María Gómez Riveros ${ }^{1}$

1. Licenciada en Español y literatura Universidad del Quindío - Maestría en Educación y Desarrollo Humano de la Universidad de Manizales.

Recibido: 10 de Noviembre del 2014

Aceptado: 26 de Enero del 2015

*Correspondencia: E-mail: amagori8710@yahoo.com

\section{RESUMEN}

El presente artículo está basado en una investigación que pretende describir las características cognitivo emotivas de los estudiantes de grado octavo de una institución Educativa de La Tebaida, Quindío, implicados como víctimas y agresores en el fenómeno del bullying. Para la obtención de la información se empleó una metodología de tipo cuantitativo - descriptivo, la información fue recolectada mediante la aplicación de cuestionarios individuales.

Palabras claves: cognición, emoción, agresor, víctima, bullying

\begin{abstract}
This article is based on a research that aims to describe the emotional cognitive characteristics of eighth grade students an educational institution of La Tebaida, Quindío, involved in the phenomenon of bullying as victims and perpetrators. For to obtain information employed a quantitative methodology - descriptive, the information was collected by applying individual questionnaires.
\end{abstract}

Keywords: cognition, emotion, aggressor, victim. 


\section{INTRODUCCIÓN}

El matoneo escolar es una problemática que se ha venido poniendo en evidencia, gracias a la amplia difusión que vienen haciendo los nuevos medios masivos de comunicación, lo que ha permitido que diversos agentes sociales acudan ante la necesidad de intervención para diagnosticar, conocer, intervenir, prevenir y tratar de erradicar dicha problemática de las instituciones educativas. Sin embargo, hasta el momento, la mayor parte de las investigaciones han estado encaminadas a la caracterización del fenómeno, el reconocimiento de sus consecuencias y el análisis de las percepciones que poseen los niños, niñas y adolescentes sobre la problemática, pero se ha dejado un poco de lado el análisis de los factores cognitivo-emotivos que caracterizan a los individuos implicados, los cuales desempeñan un papel muy importante a la hora de dar explicación a las formas de comportarnos y, por tanto, a las situaciones de matoneo escolar.

En los últimos años, diversos estudiosos (Valero, 1997, LeDoux 1999, Vallés y Vallés, 2000, Acosta 2003, Collell y Escudé 2004), han coincidido en atribuir gran importancia a la relación cognición emoción a la hora de tratar de comprender diversos fenómenos en el ser humano. Tal es el caso de Goleman (1996) quien afirma que no se puede entender la mente y sus procesos sin el mundo de las emociones. En esta misma línea Bisquerra R. (2000), Damasio A. (1996), Eich E. y otros (2003), Ortony, A., Clore, G.L. y Collins, A. (1996), coinciden en aceptar la interrelación entre los procesos cognitivos emotivos, en los que la emoción influye en la toma de decisiones y el desarrollo de procesos cognitivos al disparar recursos cognitivos y activar determinados circuitos en el cerebro lo que hace que se centre la atención en algunos asuntos y se otorgue menos importancia a otros, que a su vez influyen en la intensidad de las emociones, por lo que no pueden ser considerados como aspectos separados en los individuos sino íntimamente ligados y por tanto, de gran importancia a la hora de explicar el comportamiento de víctimas y agresores en el fenómeno del bullying.

Son múltiples las discrepancias y pocas las convergencias que se presentan a la hora de analizar los planteamientos de los diferentes teóricos sobre la emoción; existen algunos autores que para definir la emoción se basan en aspectos netamente cognitivos como el pensamiento, la evaluación y la valoración, otros, orientados por los aspectos conductuales, otor- gan mayor importancia a las expresiones faciales y motoras, como Darwin (1872/1965). Por su parte, los autores que se centran en los aspectos orgánicos, como James W. (1884/1985) y Lange C. (1984), se basan en las reacciones y cambios fisiológicos, logrando converger en la necesidad de que se evidencie un cambio y en la existencia de un estímulo que desencadena el proceso para que se dé la emoción, sin embargo, la consideración sobre el tipo de cambio y estímulos varía de acuerdo a la postura del autor.

Un aspecto de gran discusión se relaciona con la clasificación de las emociones, autores como Ekman, 1984; Izard, 1977; Tomkins, 1962, 1963; plantean la existencia de un grupo de emociones básicas que serían componentes innatos del ser humano y que van apareciendo a lo largo de su evolución, mientras otro grupo de estudiosos como Ortony y Turner (1990), Ortony, Clore y Collins (1996) plantean la imposibilidad de determinar la existencia de un grupo de emociones básicas que puedan dar origen a otras y plantean la valencia como un aspecto fundamental para jerarquizar las emociones.

\section{MATERIALES Y MÉTODOS:}

La presente investigación es de carácter descriptivo transversal. Descriptivo, ya que como lo plantean Hernández, $R$., Fernández, C., y Baptista, $P$. (2010) busca especificar las propiedades, las características y los perfiles de personas, grupos, comunidades, procesos, objetos o de un fenómeno, en este caso, el bullying, que está siendo sometido a un análisis. Transversal ya que recolecta datos en un solo momento, en un tiempo único. Su propósito es describir variables y analizar su incidencia e interrelación en un momento dado y no pretende comparar con lo sucedido en una etapa posterior. Este tipo de investigación es útil para mostrar con precisión los ángulos o dimensiones de un fenómeno, suceso, comunidad, contexto o situación.

\section{Población y muestra}

En el estudio se tuvo en cuenta una muestra inicial de 62 estudiantes de ambos sexos, con edades entre los 11 y los 17 años, pertenecientes a grado $8^{\circ}$ de una Institución Educativa de La Tebaida Quindío, los cuales fueron seleccionados de manera intencional debido a la problemática de convivencia previamente identificada y reportada por los docentes y directivos de la institución. Posteriormente, gracias a la identificación de los agresores y víctimas del grupo que se logró con 
la aplicación de un primer cuestionario, se trabajó con un grupo de 9 víctimas y 12 agresores para la identificación de sus características, de los cuales 14 eran hombres y 7 mujeres, cuyas edades se encontraban entre los 12 y los 16 años de edad, todos pertenecientes al grado octavo.

\section{Procedimiento}

\section{Fases de la investigación}

a)Búsqueda y definición de instrumentos a utilizar: Construcción del cuestionario. Una vez analizados diversos tipos de cuestionarios e instrumentos existentes relacionados con la temática manejada en la investigación, se procedió a la selección y adecuación de los cuestionarios a aplicar. Para ello se procedió a complementar el cuestionario "Bullying-Cali" con algunas preguntas que se consideraban necesarias y pertinentes, las cuales fueron sometidas a juicio de expertos externos. Posteriormente, se definieron los instrumentos que permitirían el análisis de los aspectos cognitivo emotivos en los estudiantes y que fueran acordes para nuestro contexto.

\section{b) Recogida de datos.}

Para poder obtener los datos de este estudio, en primer lugar se contactó con las directivas de la Institución Educativa con el fin de obtener su consentimiento y poder realizar el estudio. Después de la aceptación de las mismas, se realizó una reunión informativa con los estudiantes y otra con padres de familia de los estudiantes a participar, ya que son menores de edad, con el fin explicar en qué consistía el estudio, los objetivos del mismo, el alcance de la investigación, describir los instrumentos de evaluación, el número de sesiones necesarias, aclarar las posibles dudas, organizar el plan de trabajo y conseguir su consentimiento informado por escrito.

El diligenciamiento de los cuestionarios se realizó en dos sesiones (1- cuestionario para identificar agresores y víctimas y 2-cuestionarios para caracterizarlos) durante el periodo regular de clase, en el aula, de forma colectiva y contando sólo con la presencia de los investigadores. En todas las sesiones se siguió el mismo procedimiento:

- Se explicó brevemente a los alumnos el objetivo de la investigación y la importancia de su participación; se insistió en el anonimato de los datos: sus respuestas serían totalmente secretas y confidenciales.

- Se les explicaron las instrucciones para cumpli- mentar los cuestionarios indicándoles que no se trataba de un examen: no había respuestas falsas o verdaderas, ni correctas o incorrectas; no se trataba de terminar antes que el compañero, sino de hacerlo y completarlo de forma adecuada, seria y, sobre todo, sincera porque interesaba mucho su punto de vista, su opinión personal.

- Se entregó a cada uno de los estudiantes el paquete de instrumentos.

- El investigador permaneció en el aula durante toda la aplicación para resolver las dudas de los alumnos y acompañar que los cuestionarios se cumplimentaran adecuadamente.

c) Análisis de datos. Durante esta fase se realizó el tratamiento y análisis estadísticos de los datos recogidos con el paquete estadístico SPSS 15.0 versión para Windows. La fase constó de varias tareas:

- Creación de la base de datos

- Introducción de los datos

- Estudio de las propiedades psicométricas de los instrumentos

- Análisis descriptivos y frecuencias

Posteriormente, de acuerdo con los datos arrojados fue utilizado el programa de office Excel 2010 con el fin de realizar gráficos radiales los cuales permiten un fácil análisis y comprensión de los datos.

d) Informe y publicaciones. La última fase corresponde a la redacción del informe técnico en el que se da cuenta del proceso de investigación y a la publicación de artículos individuales relacionados con la temática en revistas científicas indexadas.

\section{Técnicas e instrumentos}

- Cuestionario “Bullying-Cali” Paredes M., Lega, L. y Vernon A.(2008)

Este cuestionario Tiene como objetivo identificar a los alumnos y alumnas que responden a las situaciones de Bullying (acoso y amenaza entre escolares) en condición de agresores o víctimas, y a aquellos o aquellas que no se sitúan en ninguna de las dos posibilidades.

- Escala MESSY (Traducción de Trianes y colaboradores, 2002)

Es un cuestionario formado por 62 ítems con una escala tipo Lickert con cuatro opciones (nunca, a veces, a menudo y siempre) en la que cada estudiante auto evalúa su forma de comportarse, fue diseñada para evaluar el grado de adecuación de la conducta social. 
Existen dos formatos, uno para los alumnos (de 14 a 18 años), que es la utilizada en este estudio y otro destinado a los profesores. En esta investigación se usa con el fin de identificar y comparar la manera como víctimas y agresores se auto evalúan.

En esta investigación, se ha utilizado la traducción y adaptación realizada por Trianes y colaboradores (2002)

- Cuestionario de Agresión de Buss y Perry (1992) (Adaptación de Sierra, Gutiérrez-Quintanilla et al., 2006)

El cuestionario está formado por 29 ítems en escala tipo Likert con cinco opciones (desde 1 completamente falso para mí hasta 5 completamente verdadero para mí) referidos a sentimientos y conductas agresivas. En este estudio se utilizó la versión salvadoreña de Sierra y Gutiérrez-Quintanilla (2006), compuesta por 27 ítems que conforman los cuatro factores planteados en la versión original (agresión física, agresión verbal, ira y hostilidad)

- Cuestionario ScanBullying (Almeida y Curcel, 2005)

Este cuestionario parte de una serie de láminas en las que se representan gráficamente diferentes situaciones de victimización entre iguales (físicos, verbales, exclusión social, presión de pares, etc.) en un contexto escolar. Este instrumento fue creado con el fin de explorar las representaciones de los preadolescentes y adolescentes acerca de diferentes aspectos del maltrato entre iguales en el contexto escolar como: percepciones sociales, significados, actitudes, atribuciones causales, sentimientos, emociones morales y razonamiento sociomoral.

En esta investigación, este instrumento se usa con el fin de analizar la manera cómo las víctimas caracterizan a los agresores y al contrario, cómo los agresores caracterizan a las víctimas

\section{RESULTADOS Y DISCUSIÓN}

Con respecto a las manifestaciones más específicamente cognitivas, en el estudio realizado con estudiantes de grado octavo de una Institución Educativa pública de La Tebaida Quindío, se encontró que las víctimas cuentan con un mayor grado de análisis y reflexión sobre los acontecimientos, sus causas y consecuencias y, por lo tanto, poseen un mejor control sobre sus emociones, mientras que los agresores suelen evadir dichas tareas y responder de manera más instintiva. Esto último coincide con las conclusiones de diferentes teóricos que plantean que los agresores son impulsivos, con escaso autocontrol en sus relaciones sociales, falta de habilidades sociales, baja tolerancia a la frustración, bajo rendimiento escolar, conductas desafiantes y dificultad para cumplir normas en el ámbito educativo (Cerezo, 2012, Collell y Escudé, 2004; Díaz-Aguado, 2002)

Al referirnos al tema de la causalidad adjudicada (producto de la valoración que hacen los actores), se evidenció que las víctimas, en un alto porcentaje, consideran que estas situaciones se dan porque son propias de la edad, y como situaciones de diversión para un grupo (agresores).Los agresores, por su parte, consideran que estas son situaciones que se dan por hacer bromas a sus compañeros, sin embargo, al cuestionarles sobre la finalidad, manifestaron que buscan dominar al otro y atribuyeron como causa de su conducta, la educación recibida en casa, consideraciones totalmente opuestas a las de las víctimas.

Dado lo anterior, es posible constatar que se concibe diferente según el rol en la conducta del bullying, sin embargo, es de destacar que el hecho de que las víctimas estén atribuyendo como causas de las agresiones, factores como la edad y la diversión, es algo que preocupa a los investigadores, educadores y padres de familia, ya que de alguna manera permite justificar las agresiones y lleva a indagar sobre el grado de naturalización que se le está otorgando a la violencia en este contexto.

Con relación a las manifestaciones asociadas a los procesos emotivos, fue posible constatar que los agresores, en su mayoría manifiestan que disfrutan y se sienten alegres, satisfechos o poderosos, ante las situaciones de agresión que protagonizan, lo cual concuerda con lo planteado por Menesini y otros (1999) quien afirmó que los matones no se sienten culpables por sus acciones, y a menudo experimentan indiferencia y orgullo delante de sus iguales, de tal manera, es posible afirmar que los agresores, a pesar de manifestar o caracterizar a las víctimas de manera positiva, al indicar que son personas humildes, inteligentes, deseables como amigos, buenas personas, educadas, sinceras, es evidente que no están manejando sentimientos empáticos hacia ellas y, por el contrario, están centrándose en la caracterización de los aspectos menos positivos de las víctimas tales como que son 
tímidos, débiles y aburridos, según su percepción, como base para crear la imagen mental que poseen de las víctimas y que les permite fortalecer su conducta como agresor.

En relación con lo anterior, se encontró que la mayoría de los agresores $(82 \%)$ no se considera una persona agresiva y el porcentaje restante atribuyen como causa de "ser agresivo" situaciones aprendidas del contexto o un acontecimiento que marcó su vida en determinado momento.

Con referencia a las emociones que más prevalecen en agresores y víctimas es posible afirmar que los agresores suelen manifestar en su mayoría, alegría, orgullo, satisfacción, poder y rabia. Las víctimas, suelen experimentar preocupación, tristeza, vergüenza y rabia. Esta última, es una de las emociones más comunes tanto en víctimas como agresores, sin embargo, es posible plantear que los agresores hacen uso de ésta como parte de la agresión e intimidación hacia las víctimas, mientras que estas últimas la manifiestan como resultado de su impotencia y como posible mecanismo de defensa.

En la siguiente tabla, se presentan las principales emociones halladas en víctimas y agresores:

Tabla 1. Emociones según el rol

\begin{tabular}{ll}
\hline \multicolumn{1}{c}{ AGRESORES } & \multicolumn{1}{c}{ VÍCTIMAS } \\
\hline Alegría & Preocupación \\
Orgullo & Tristeza \\
Satisfacción & Vergüenza \\
Poder & Rabia \\
Rabia & \\
Tabla l: elaborada por la autora &
\end{tabular}

Al abordar la pregunta sobre la frecuencia de la culpabilidad que experimenta el agresor, las víctimas consideraron que el agresor siempre se siente culpable ante lo que realiza, sin embargo, al analizar las respuestas dadas por los agresores, ellos afirmaron todo lo contrario; manifestaron que nunca se sienten culpables, en porcentajes que se presentan en los siguientes gráficos:

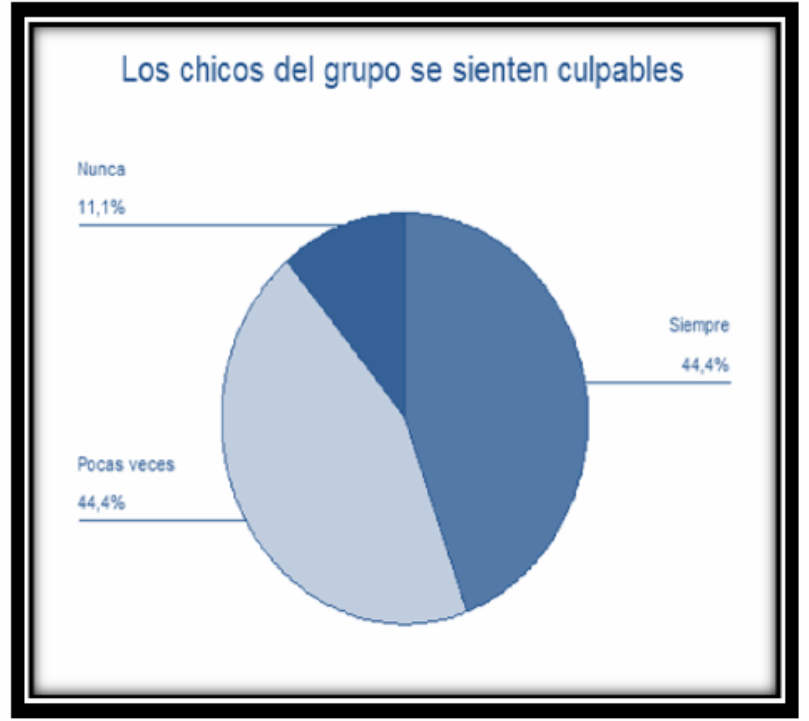

Figura 1 Opinión de víctimas sobre sentimiento de culpa en agresores

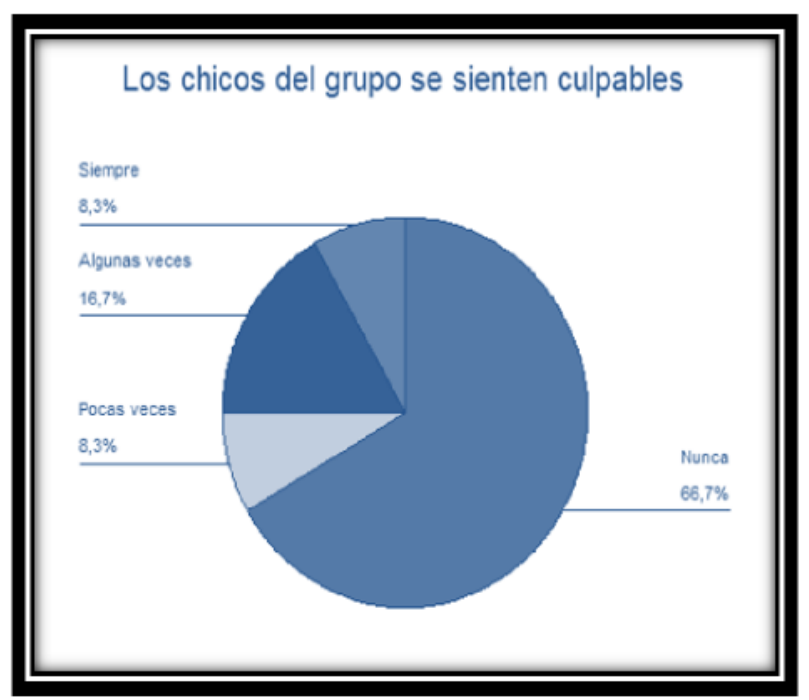

Figura 2 Opinión de agresores sobre sentimiento de culpa en agresores.

Esto mismo fue mencionado por Smorti y Ciucci (2000), al plantear que víctimas y agresores difieren en la manera de interpretar la realidad social. De esta manera, las visiones diferentes, no se corresponden con una imagen distorsionada o errónea, o con interpretaciones ciertas o falsas, sino que llevan a explicaciones diferentes sobre el matoneo escolar de acuerdo con la experiencia vivida por cada uno de los actores. Al centrarnos en la caracterización personal que hacen de sí mismas las víctimas, encontramos que se re- 
conocen como personas solitarias, introvertidas, agradecidas, en ocasiones temerosas y que se entristecen cuando ofenden o hacen daño a alguien, en contraste con el perfil de los agresores quienes se reconocen como personas alegres, extrovertidas, que nunca permanecen ni juegan solos, no temen hablar en público y que han llegado a estar muy furiosos como para romper algo.

Tabla 2 Autodefinición de agresores y víctimas

\begin{tabular}{ll}
\hline \multicolumn{1}{c}{ AGRESORES } & \multicolumn{1}{c}{ VÍCTIMAS } \\
\hline Personas alegres & Personas solitarias \\
Extrovertidas & Introvertidas \\
Nunca permanecen ni jue- & Agradecidas \\
gan solos & \\
No temen hablar en público & En ocasiones temerosas \\
Han llegado a estar muy & Se entristecen cuando ofen- \\
furiosos como para romper & den o hacen daño a alguien. \\
algo. & \\
\hline
\end{tabular}

Tabla 2: Elaborada por la autora

Lo anterior permite confirmar lo planteado por Olweus (1998) sobre algunas características de agresores y víctimas, entre las que propuso a las víctimas como personas inseguras, temerosas, sumisas y con pocos amigos y a los agresores como personas agresivas, con poco control de la ira, seguros de sus acciones y más populares que las víctimas.

Para terminar, es importante anotar que tanto las víctimas como los agresores, consideran que las situaciones de matoneo no son algo nuevo sino que vienen desde siempre y peor aún, que van a continuar por siempre (ver gráficos 3 y 4 ), lo que permite deducir que no se están evidenciando acciones orientadas a enfrentar y disminuir la problemática en la institución educativa o que éstas se han naturalizado de tal manera que se han llegado a considerar como parte de la cotidianidad.

\section{CONCLUSIONES}

La escuela como agente importante en los procesos de formación y socialización de niños y jóvenes juega un papel fundamental en la adquisición de hábitos y maneras de interactuar de sus estudiantes. Si bien la Institución Educativa desempeña un importante papel en la formación académica de los individuos, también influye de manera decisiva en la formación del carácter y la personalidad de los que allí conviven, dadas las características y los tipos de interacción entre estudiantes y entre estudiantes y docentes y el manejo

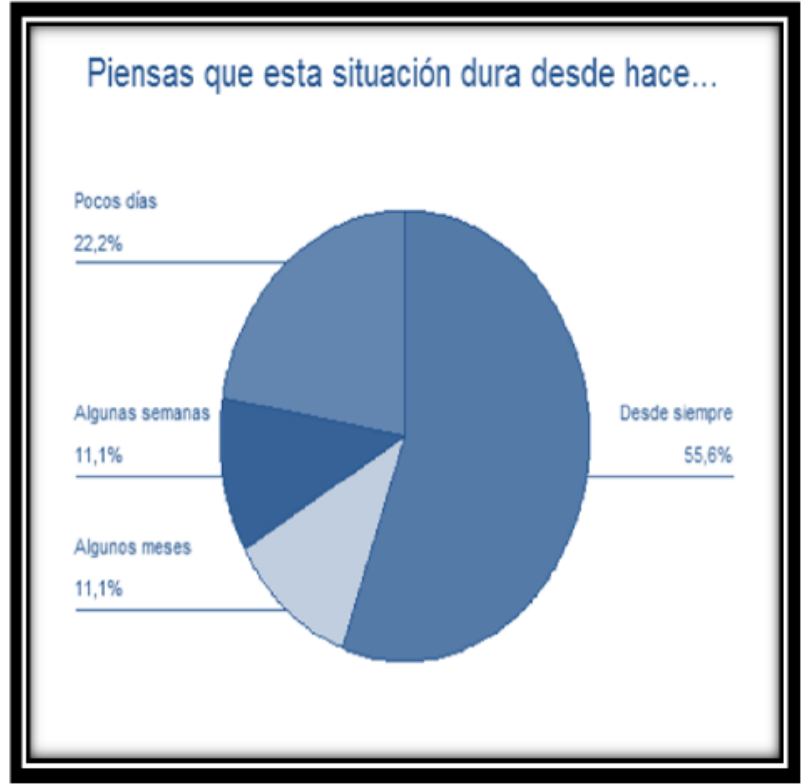

Figura 3 Opinión sobre la trayectoria de las situaciones de bullying

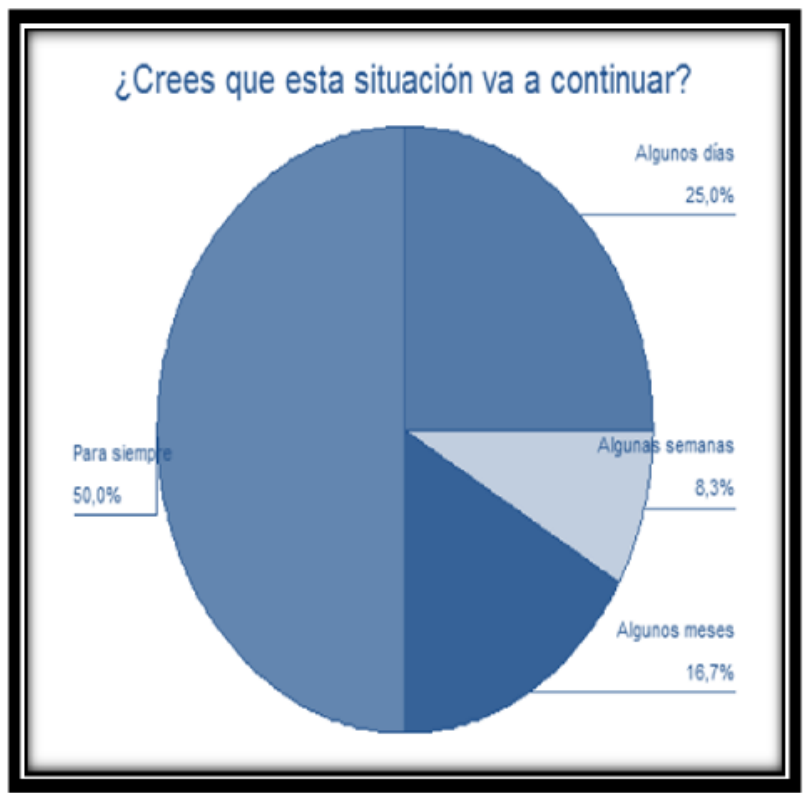

Figura 4 Opinión sobre la posible continuidad de las situaciones de bullying

otorgado a las relaciones de poder que allí surgen, los estudiantes formarán determinados conceptos y percepciones acerca de la manera en que deben ser manejadas las relaciones interpersonales, en este caso, con sus pares.

En este sentido, y al conocer la problemática de bullying presente en la institución educativa, cobra gran importancia la formación de los estudiantes en los aspectos relacionados con los procesos cognitivo - 
emotivos del individuo, los cuales están implicados en todas las situaciones y vivencias que a diario se presentan en la escuela al requerir de procesos como la percepción, la toma de decisiones, el análisis y valoración de situaciones, el reconocimiento y control de las emociones propias y de los demás, entre otros $\mathrm{y}$, por tanto, su adecuado manejo y conocimiento puede contribuir a hacer frente a las situaciones de bullying detectadas en la institución. López F. María (2008)afirma:

Laeducación en la emociónyla sensibilidadmodificanosóloel carácter, sino elmodo decomprender elmundoyla capacidad cognitivade enfrentarse aél. La emoción es también, cognición. Y que, en la toma de decisiones y planificación del futuro, ambos son indisociables. $p$ (224)

Dada la importancia que representa conocer y tener conciencia sobre los procesos cognitivo emotivos propios y de los otros para establecer mejores relaciones interpersonales gracias a la autorregulación, se considera pertinente mencionar algunos aspectos que podrían tenerse en cuenta con el fin de mejorar las relaciones interpersonales entre los estudiantes, hacer frente a la problemática del bullying y promover una formación personal en la que se incluyan los procesos cognitivo emotivos y metacognitivos, de tal manera que la comunidad educativa cuente con recursos y estrategias conscientes para afrontar diversas situaciones en su vida cotidiana.

Estas estrategias, pueden ser:

- Realizar un análisis del contexto para identificar necesidades emocionales que deben ser atendidas, a través de la implementación de diversas estrategias, enfocadas a la educación emocional, que redunden en el mejoramiento de la convivencia escolar y formación personal de la comunidad educativa.

- Diseñar un programa que fomente el desarrollo de las competencias emocionales en los miembros de la comunidad educativa y que pueda ser implementado a través de los diferentes proyectos institucionales y el área de educación emocional creada en la institución, para, de esta manera, brindar apoyo a los agresores, de tal forma que puedan aprender a analizar objetivamente la información relacionada con las relaciones interpersonales para lo cual es. Para lo anterior, es necesario que sean capaces de realizar diversos procesos cognitivo emotivos para analizar las causas, consecuencias y alternativas existentes frente a cualquier situación que se presente con otra(s) persona(s), además, que sean puedan de reconocer sus emociones y las de los demás, expresarlas de adecuadamente y regularlas, y, de este modo, favorecer el desarrollo de la empatía y las conexiones afectivas y cognitivas con los demás.

En el caso de las víctimas, el apoyo desde el programa podría estar orientado al fortalecimiento de su autoestima y al desarrollo de estrategias de afrontamiento frente al mal trato recibido, que les permita fortalecer su auto imagen y auto confianza, y desarrollar un mayor grado de control de pensamientos y emociones negativas generados por ellas mismas.

Por otra parte, es importante que tanto víctimas como agresores aprendan el manejo de estrategias metacognitivas, de autocontrol y auto evaluación que les permita reconocer cuáles son sus reacciones, emocionales, cognitivas y físicas, más recurrentes en las situaciones de conflicto, y poner en práctica comportamientos alternativos que les permitan afrontar de mejor manera situaciones de conflicto.

- Brindar formación a los docentes, padres de familia y estudiantes sobre los procesos cognitivos emotivos que están implicados en el desarrollo de actividades de la vida cotidiana, de tal manera que se promueva la necesidad de comprender de una manera renovada la concepción sobre las emociones y su papel en el diario vivir.

- Propiciar ambientes y condiciones que promuevan la motivación en los estudiantes para que las clases de educación emocional sean asumidas como algo agradable e importante en su vida, no como una asignatura de menor importancia como sucede en la actualidad. 


\section{BIBLIOGRAFIA}

- Acosta, A. (2003). Resolución de conflictos y regulación de sentimientos. En A. Muñoz, B. Molina y F. Jiménez Actas del I Congreso Hispanoamericano de Educación y Cultura de Paz (pp. 293-304). Granada: Universidad de Granada.

- Almeida, A. y Curcel, M. (2005) Cuestionario Scan Bullying versión portuguesa y castellana. Registro de obra en inspección general de la actividades culturales. Ministerio de cultura.

- Bisquerra, R. (2000). Educación emocional y bienestar. Barcelona: Praxis.

- Cerezo R. F. (2012) Bullying a través de las TIC. Boletín Científico Sapiens Research Vol. 2(2) pp: $24-29$

- Collell, J., y Escudé, C. (2004), Rol de les emocions en elsprocessos de maltractament entre alumnes. Àmbits de Psicopedagogia, 12, 21-26.

- Damasio, A. R. (1996). El error de Descartes. Barcelona: Crítica.

- Darwin, C.R. (1872/1965). The Expression of the Emotions in Man and Animals.Chicago: University of Chicago Press.

- Díaz-Aguado, M. J. (2002). Convivencia escolar y prevención de la violencia. Madrid: Publicaciones del Ministerio de Educación y Ciencia, CNICE

- Eich E. y otros (2003) Cognición y emoción. Bilbao: Desclee De Brouwer,

- Ekman, P. (1984). Expression and the nature of emotion.En K.S. Scherer y P. Ekman (Eds.), Approaches to emotion (pp. 319-344).Hillsdale, NJ: Lawrence Erlbaum Associates.

- Goleman, D. (1996). La inteligencia emocional. Barcelona: Kairos.

- Hernández, R., Fernández, C., Baptista, P. (2010) Metodología de la Investigación. 5ta Edición. México , México D.F.: Editorial McGraw Hill.

- Izard, C. E. (1994). Innate and universal facial expressions: Evidence from development and cross-cultural research. Psychological Bulletin, 115 (2), 288-299.

- Izard, C.E. (1977). Human emotions. New York: Plenum Press.

- James, W. (1884/1985). What is an emotion?.En E. Gaviria (traduct), Estudios de Psicología, 21, $57-73$

- Lang, P. J. (1984). Cognition in emotion: Concept and Action. En C. E. Izard, J. Kagan y R. B. Zajonc (Eds.), Emotions, Cognitions and Behaviour.Cambridge: Cambridge UniversityPress.

- LeDoux, J. (1999). El cerebro emocional. Barcelona: Ariel-Planeta.

- López F. M. A. (2008) Cognición y emoción: el derecho a la experiencia a través del arte. Pulso $\mathrm{N}^{\circ} 31$, pp. 221-232

- Menesini, E., Fonzi, A., Ciucci, E., Almeida, A., Ortega, R., Lera, M.J., Costabile, A., Lo Feudo, G., H. Cowie, S. Ferrazzuolo y C.del Barrio (1999).Bullying and emotions. En Nature and Prevention of Bullying, TMR Network Project: http://www.gold.ac.uk/tmr/reports/aim2_firenze2. html (Consultado 25/07/2012).

- Olweus, D. (1998). Conductas de acoso y amenaza entre escolares. Madrid, España: Ediciones Morata

- Ortony, A. y Turner, TJ. (1990): What's basic about basic emotions? Psychological Review, 97, 315-331.

- Ortony, A., Clore, G. L. y Collins, A. (1996). La Estructura Cognitiva de las Emociones. Madrid: SigloVeintiuno.

- Paredes, M., Álvarez, M., Lega, L. \& Vernon, A. (2008). Estudio exploratorio sobre el fenómeno del "bullying" en la ciudad de Cali, Colombia. Revista Latinoamericana de Ciencias Sociales, Niñez y Juventud, 6 (1), 295-317 
- Sierra, J. C., Gutiérrez-Quintanilla, R. y Delgado-Domínguez, C. J. (2006). Estudio psicométrico de la versión española del Cuestionario de Agresividad de Buss y Perry en población universitaria salvadoreña. Manuscrito sometido a publicación.

- Smorti, A., \&Ciucci, E. (2000).Narrative strategies in bullies and victims. Aggressive Behavior, $26,33-48$

- Tomkins, S.S. (1962). Affect, Imagery, Consciousness. The negative affects (Vol. 1). New York: Springer.

- Tomkins, S.S. (1963). Affect, Imagery, Consciousness. The negative affects (Vol. 2). New York: Springer.

- Trianes M.V, Blanca M.J, Muñoz A., García B., Cardelle Elawar M. y Infante L. (2002). Relaciones entre evaluadores de la competencia social en preadolescentes: Profesores, iguales y autoinformes. Anales de Psicología. Vol. 18, 2, 197- 214.

- Valero, L. (1997): La evaluación del comportamiento infantil: características y procedimientos. Apuntes de Psicología, 51 pp.51-79.

- Vallés, A. y Vallés, C. (2000). Inteligencia emocional. Aplicaciones educativas. 